\title{
INFLUENCE OF ABSORPTION DUE TO COMPETING PROCESSES ON PERIPHERAL REACTIONS
}

\author{
K. Gottfried, J.D. Jackson, B. Svensson \\ CERN, Geneva
}

(Presented by M. ROSS)

Quasi-two-body peripheral reactions at a few $\mathrm{GeV}$, involving the production of one or more unstable particles or resonances, are discussed in terms of the one-meson-exchange model, modified to include the absorptive effects of competing processes. The specific formalism, patterned after the original work of Sopkovich [1], has been described in detail elsewhere [3]. The spins of the particles are included in a consistent manner, enabling us to discuss the decay correlations of the resonances as well as the production angular distributions. In Ref. [2] application of the model was made to the reaction $\pi^{-} p \rightarrow Q^{-} p$ at $4 \mathrm{GeV} / \mathrm{c}$ incident momentum. The theoretical production cross section was found to be in good agreement with the data [3], in both angular dependence and in absolute value. In the present paper the model is applied to the o-production reaction at other energies and to the $K^{+} p \rightarrow K^{*} p$ and $K^{*} p \rightarrow K^{\circ} N^{*}$ reactions at $3 \mathrm{GeV} / \mathrm{c}$ [4].

\section{1. o-PRODUCTION}

The cross section and decay correlations for the reaction $\pi^{ \pm} p \rightarrow \mathrm{Q}^{ \pm} p$ has been calculated as a function of incident momentum from 1.6 to $4 \mathrm{GeV} / \mathrm{c}$ assuming one pion exchange as the mechanism. The absorptive effects are deduced from the elastic $\pi^{ \pm} p$ scattering data at each energy. Since the coupling constants are known $\left(g_{\rho \pi \tau}^{2} / 4 \pi=2.0\right.$ for $\Gamma_{\rho}=$ $\left.=100 \mathrm{MeV}, G^{2} p p \pi^{\circ} / 4 \pi=14.5\right)$ there are no adjustable parameters in the calculation. The calculated angular distributions of production are in generally good agreement with available data. The comparison at $4 \mathrm{GeV} / \mathrm{c}$ for $\pi^{-}$is given in Ref. [2]. The data at 1.59 $\mathrm{GeV} / \mathrm{c}$ [5] for $\pi^{-}$are compared with the theory in the top half of Fig. 1. Similar agreement occurs at other energies.

The total cross section for $\varrho$-production is shown as a function of incident momentum in the bottom half of Fig. 1. The difference between the theoretical curves for $\pi^{+}$and $\pi^{-}$ is due to the differences in the $\pi^{+} p$ and $\pi^{-} p$ elastic diffraction scattering (which determines the absorption). This difference is largest in the neighbourhood of $2 \mathrm{GeV} / \mathrm{c}$. The agreement between theory and experiment cannot be said to be excellent, but the general energy dependence seems roughly the same and the $\pi^{+}$cross sections appear to be larger than the $\pi^{-}$cross sections, as expected theoretically. Two remarks can be made. Firstly, the theoretical description breaks down at low energies where not a large number of partial waves are involved and the outgoing center-of-mass momentum is much different from the incoming momentum. The theoretical curves are beginning to become unreliable below $2.0 \mathrm{GeV} / \mathrm{c}$. Secondly, the experimental problems involved in determining an absolute cross section for production of a resonance (usually sitting on top of a sizable background) are not trivial. Theorists may therefore double, at least in their minds eye, the errors on the points when looking at Fig. 1.

The decay correlation data can be expressed in terms of the elements $\varrho_{00}, \varrho_{1,-1}$ and Re $\varrho_{10}$ of the $\mathrm{Q}$-meson's spin density matrix [10]. The simple one-pion-exchange model gives $\varrho_{00}=1$, $\varrho_{1,-1}=0=\operatorname{Re}_{10}$. With absorption the theoretical values depart from these simple predictions, especially at large production angles, as shown at $4 \mathrm{GeV} / \mathrm{c}$ in Fig. 5 of Ref. [2]. The experimental average values for $4 \mathrm{GeV} / \mathrm{c}$ $\pi^{+}$from Ref. [8] are $\left\langle Q_{00}\right\rangle=0.70 \pm 0.08$, $\left\langle\varrho_{1,-1}\right\rangle=0.17 \pm 0.14, \quad\left\langle\operatorname{ReQ}_{10}\right\rangle=-0.074 \pm$ \pm 0.070 , in reasonable agreement with the theoretical members $0.65,0.06$ and -0.18 , respectively.

\section{2. $K^{*}$ PRODUCTION AT $3 \mathrm{GeV} / \mathrm{c}$}

The CERN data [4] on decay correlations of the $K^{*}$ produced in the reaction $K^{+} p \rightarrow$ $\rightarrow K^{\circ} \pi+p$ indicate predominantly vectors 

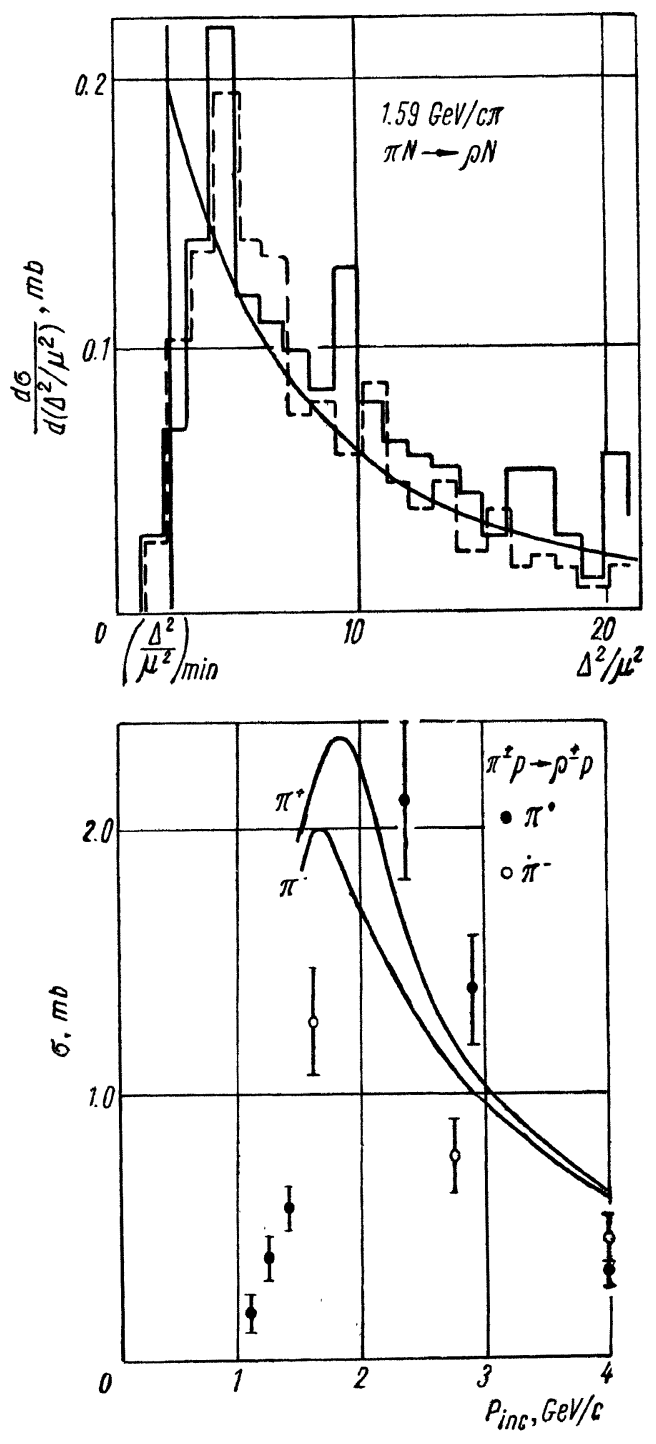

Fig. 1. Q-production. T o p: Comparison of the theoretical and experimental distributions for $\pi^{-} p \rightarrow 0$ $\mathrm{N}$ at $1.59 \mathrm{GeV} / \mathrm{c}$. The smooth curve is theoretical and has arbitrary normalization. The solid histogram represents the data on $\pi^{-} p \rightarrow Q^{-} p$; the dashed histogram is one half of the cross section for $\pi^{-} p \rightarrow Q^{0} n$. The data are from Fig. 19 of Ref. [5]. B o t t o m: Total cross section for the reaction $\pi p \rightarrow \varrho p$ as a function of incident momentum. The theoretical curves are actually the cross section for production into centre of-mass angles with $1.0 \leqslant \cos \theta \leqslant 0.7$, representing the bulk of the cross section and certainly its peripheral part. The lowest three $\pi^{+}$points are from Ref. [6]. The $\pi^{+}$values at $2.34-2.90 \mathrm{GeV} / \mathrm{c}$ are from Ref. [7]; the $\pi^{+}$point without errors at $4 \mathrm{GeV} / \mathrm{c}$ is from Ref. [8]. The $\pi^{-}$cross sections are from Ref. [5] $(1.59 \mathrm{GeV} / \mathrm{c})$, Ref. [9] $(2.75 \mathrm{GeV} / \mathrm{c})$, and Ref. [3] $(4 \mathrm{GeV} / \mathrm{c})$.

meson exchange when interpreted in terms of the simple peripheral model [11]. The angular distribution of production and decay correlations have been calculated with the present model using the elastic diffraction scattering data [12] to infer the absorption. A mixture of pion exchange and vector meson exchange has been taken. Purely vector meson exchange gives much too broad an angular distribution, although good agreement is of course found for the decay correlations. The relative amounts of pion and vector meson ( $\varrho$ or $\omega)$ exchange, their relative sign, and the ratio of tensor to vector couplings in the vector meson exchange, were varied in an attempt to fit the absolute cross section, its angular dependence and the decay correlations. The pion exchange diagram is fixed in absolute magnitude by the $K^{*}$ width of $45 \mathrm{MeV}$ and
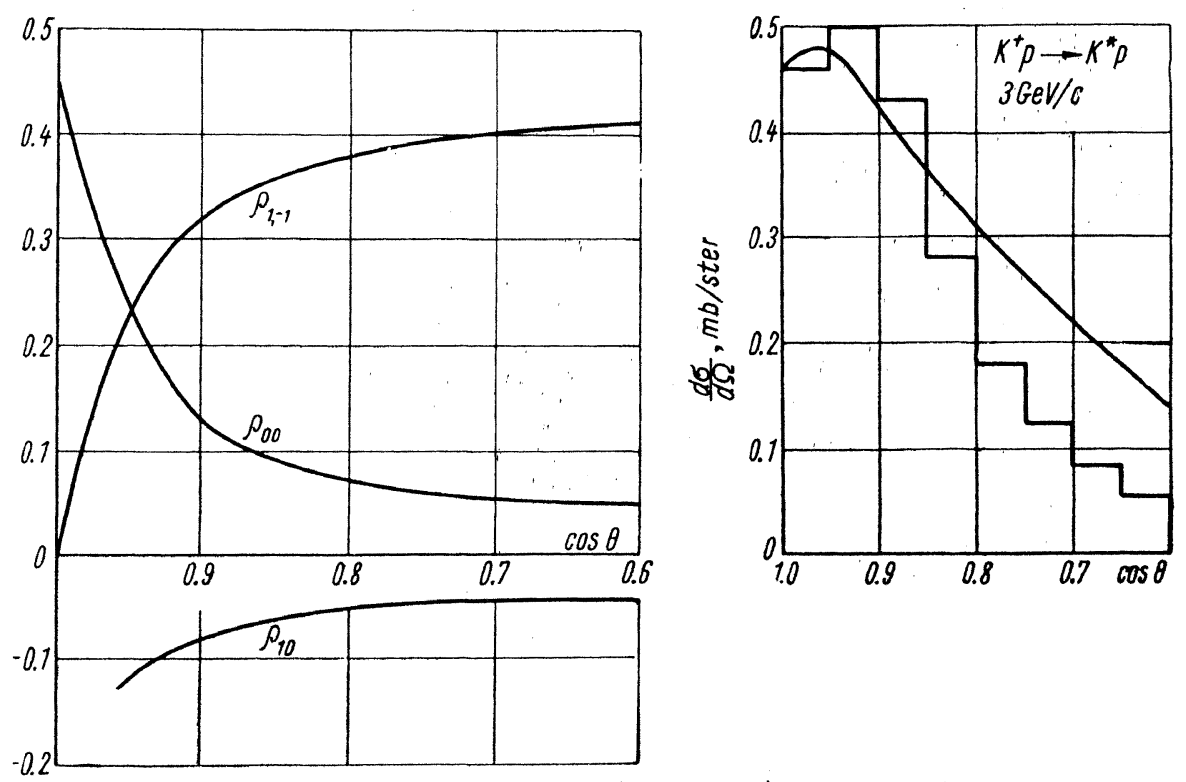

Fig. 2. $K^{*}$ production in $K^{+} p \rightarrow K^{0} \pi^{+} p$ at $3 \mathrm{GeV} / \mathrm{c}$. 

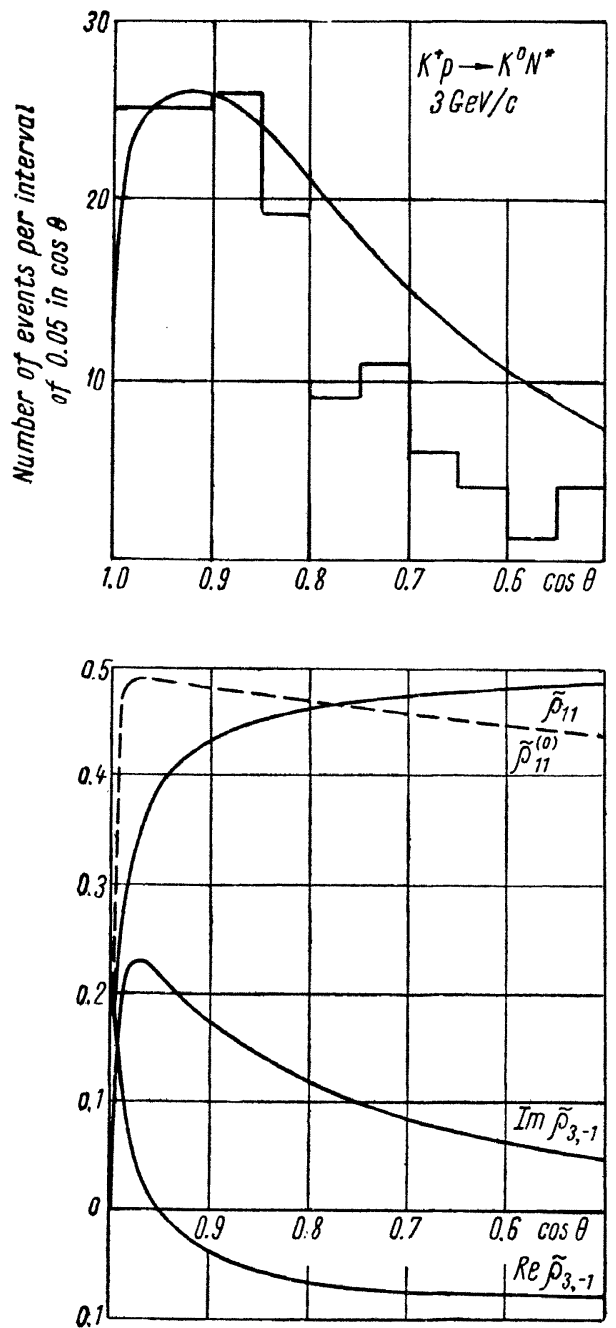

Fig. 3. $N^{*}$ production in $K^{+} p \rightarrow K^{0} \pi^{+} p$ at $3 \mathrm{GeV} / \mathrm{c}$. T o p: Angular distribution of production. The theoretical curve is normalized to the data at the peak. $\mathrm{B}$ o t $\mathrm{t}$ o m: $\mathrm{N}^{*}$ density matrix elements relative to the normal to the production plane. The dashed curves is that of the Q-exchange model of Ref. [11]. The solid curves are for the same model, with absorptive effects. The Stodolsky - Sakurai model [13] gives $\varrho_{11}=0.5, \varrho_{3},-1=0$ at all angles.

the known $\pi N$ coupling constant; this provides a severe restriction on the allowable variations in the vector meson exchange amplitude. A possible fit is shown in Fig. 2, where the theoretical angular distribution is compared with the data [4] and the elements of the $K^{*}$ density matrix are shown. The coupling constants are

$$
\begin{gathered}
g^{2} K^{+} \pi^{0} K^{*} / 4 \pi=0.75, \quad G^{2} p p \pi^{0} / 4 \pi=14.5, \\
\frac{G_{v}^{2}}{4 \pi} \times \frac{f^{2}}{4 \pi}=11.3, \quad G_{T}=-0.07 G_{v} .
\end{gathered}
$$

where the notation is that of Ref. [11]. The relative sign of the two amplitudes is such that they interfere destructively in the forward direction.
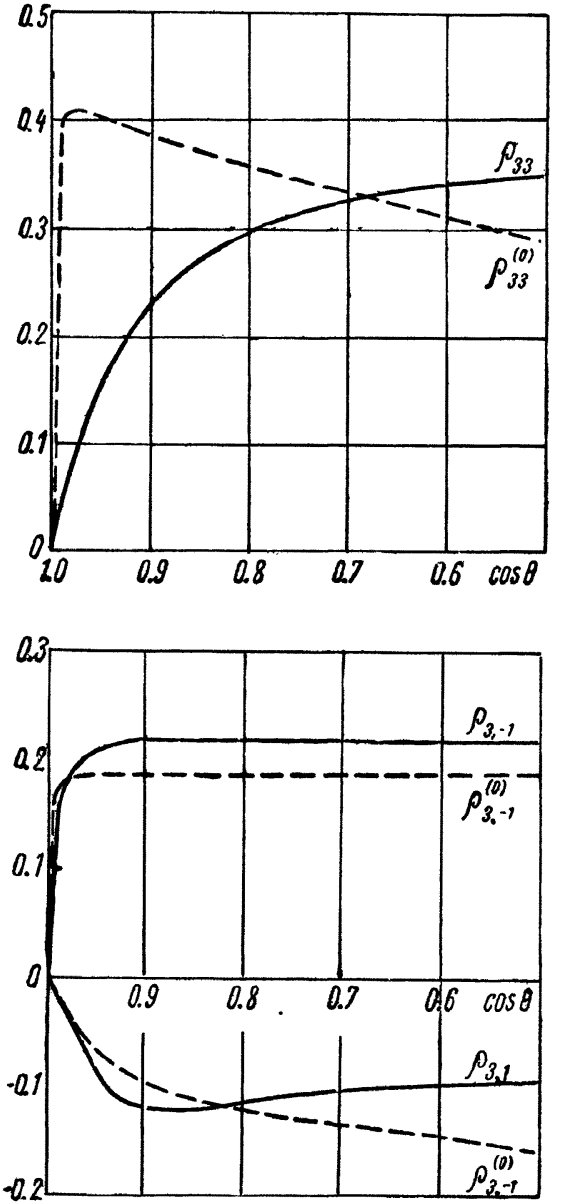

Fig. 4. The canonical density matrix elements for $N^{*}$ in the same reaction as Fig. 3 . The solid and dashed curves have the same meaning as in Fig. 3. The Stodolsky - Sakurai model gives $\varrho_{33}=0.375, \varrho_{3},-1=$ $=0.216, \varrho_{3,1}=0$.

The angular distributions are qualitatively similar, although the experimental one seems more sharply peaked than the theoretical one. The density matrix elements reflect the mixture of pion and vector exchanges; for example, $\varrho_{00}$ has a sizable value at zero angle where pion exchange is most important and fall towards zero at large angles, as is appropriate for vector exchange. Preliminary analysis of the CERN data as a function of angle gives experimental density matrix elements which show the same trends as in Fig. 2. The average values, $\left\langle\varrho_{00}\right\rangle=0.07 \pm 0.06, \quad\left\langle\varrho_{1},-1\right\rangle=0.32 \pm 0.06$, $\left\langle\operatorname{Re} \varrho_{10}\right\rangle=-0.10 \pm 0.05$, already published [4], are in reasonable accord with the theoretical averages of $0.13,0.31$ and -0.07 , respectively. 


\section{3. $N^{*}$-PRODUCTION IN $K^{+} p$ AT $3 \mathrm{GeV} / \mathrm{c}$}

Figures 3 and 4 show the theoretical results calculated with our model for the reaction $K^{+} p \rightarrow K^{\circ} N^{*}$ at $3 \mathrm{GeV} / \mathrm{c}$, assuming the mechanism is the o-exchange model of Stodolsky and Sakurai [13]. Figure 3 gives a comparison of the theoretical and experimental angular distributions and the theoretical $N^{*}$ density matrix with respect to the normal to the production plane as $z$-axis. In the angular distribution there is evidence that the data are more peripheral than expected from the theory. The experimental decay correlations [4] relative to the normal give $\left\langle\varrho_{11}\right\rangle=0.45 \pm 0.06$, in good agreement with the theoretical results in Fig. 3.

The density matrix elements shown in Fig. 4 are those with respect to the incident nucleon as $z$-axis, as seen in the $N^{*}$ rest frame. The experimental average values [4] are $\left\langle\varrho_{33}\right\rangle=$ $=0.28 \pm 0.6, \quad\left\langle\operatorname{Re} \varrho_{3},-1\right\rangle=0.21 \pm 0.05$, $\left\langle\operatorname{Re}_{3,1}\right\rangle=-0.04 \pm 0.65$, as compared with the theoretical averages of $0.27,0,21$ and -0.10 , respectively.

\section{REMARKS}

The comparison of theory and experiment on $\pi N \rightarrow \mathrm{\varrho} N, K N \rightarrow K^{*} N$, and $K N \rightarrow K N^{*}$ reactions in the few $\mathrm{GeV} / \mathrm{c}$ range presented here shows that the peripheral model, modified to include absorptive effects due to competing processes, can give a reasonable account of the absolute value of the cross sections, the angular distributions of production, and the decay correlations. The absorption of the low partial waves provides a collimation which accounts well for the momentum transfer dependence previously attributed to ad hoc form factors, without destroying beyond recognition the angular correlations of the meson exchange model.

The model works least well (as far as the angular distributions of production are concerned) in reactions involving $N^{*}$ production. This is shown in $F$ ig. 3, where the theoretical distribution for $K^{+} p \rightarrow K^{\circ} N^{*}$ at $3 \mathrm{GeV} / \mathrm{c}$ is seen to be broader than experiment, and also occurs for the analogous reaction $\pi^{+} p \rightarrow \pi^{\circ} N^{*}$ at $4 \mathrm{GeV} / \mathrm{c}$ [14] (not shown here), and in the $K^{+} p \rightarrow K^{*} N^{*}$ at $3 \mathrm{GeV} / \mathrm{c}$ [4] (not shown here). It appears, that it is necessary to include the $N^{*}$ spin in an explicit manner in evaluating the absorptive effects and(or) to be more realistic in treating the $N^{*}$ as a broad resonance, rather than a slightly unstable particle.

\section{REFERENCES}

1. Sop k o v i c h N. J. Nuovo cimento, 26, 186 (1962). Recent applications have been made by $D$ u r a nd L. and $C h$ i u Y. T. Phys. Rev. Lett., 12, 399 (1964) and R o s s M. H., S h a w G. L. Phys. Rev. Lett., 12, 627 (1964). A somewhat oversimplified version has been discussed by $\mathrm{D}$ a $\mathrm{r} A$. et al., Phys. Rev. Lett., 12, 82 (1964) and D a r A., T o b o c m a n W. Phys. Rev. Lett., 12, 511 (1964).

2. Got t f r i e d K. and J a c k s o n J. D. Nuovo cimento (in press); GERN, Preprint TH 428 (21 May, 1964).

3. Aachen - Birmingham - Bonn - Hamburg London - München collaboration. Nuovo cimento, 31, 729 (1964).

4. L y n c h G. R. et al. Phys. Lett., 4, 359 (1964); Ferro-Luz i $M$. et al. Proc. 1963 Sienna International Conference on Elementary Particles, Vol. 1, p. 189 (Sept. 30-Oct. 5, 1963); and private communication.

5. Saclay-Orsay - Bari - Bologna collaboration. Nuovo cimento, 29, 513 (1963).

6. F o e $1 \mathrm{~s} \mathrm{c}$ h e $\mathrm{H}$. et al. Proc. $1962 \mathrm{CERN}$ International Conference on High Energy Physics, Geneva, 1962, p. 36.

7. A $1 \mathrm{f} \mathrm{f}$ C. et al. Phys. Rev. Lett., 9, 322 (1962).

8. Aachen - Berlin - Birmingham - Bonn Hamburg - London - München collaboration. Paper submitted to this conference.

9. Saclay - Orsay - Bari - Bologna collaboration. Preprint, April, 1964.

10. Got tfried $K$. and $J$ a ckson J. D. Nuovo cimento (in press). CERN, Preprint TH 397 (15 January, 1964).

11. J a c ks o n J. D. and $P \mathrm{i} 1 \mathrm{k} \mathrm{uh} \mathrm{n} \mathrm{H.} \mathrm{Nuovo}$ cimento (in press). CERN, Preprint TH 409 (4 March, 1964).

12. D e b a i s i e ux J. et al. Paper submitted to this conference.

13. Stodolsky L. and $\mathrm{Sakurai} J$. J. Phys. Rev. Lett., 11, 90 (1963); S t o d o $1 \mathrm{~s} \mathrm{k} \mathrm{y} \mathrm{L.}$ Phys. Rev., 134, B1099 (1964); see also Ref. [11].

14. Aachen - Berlin - Birmingham - Bonn Hamburg - London - München collaboration. Phys. Lett., 10, 229 (1964). 